\title{
REPRESENTATION THEOREMS FOR COMPACT OPERATORS
}

\author{
DANIEL J. RANDTKE
}

\begin{abstract}
It is shown that $c_{0}$ (the Banach space of zeroconvergent sequences) is the only Banach space with basis that satisfies the following property: For every compact operator $T: c_{0} \rightarrow E$ from $c_{0}$ into a Banach space $E$, there is a sequence $\lambda$ in $c_{0}$ and an unconditionally summable sequence $\left\{y_{n}\right\}$ in $E$ such that $T \mu=\sum \lambda_{n} \mu_{n} y_{n}$ for each $\mu$ in $c_{0}$. This result is then used to show that a linear operator $T: E \rightarrow F$ from a locally convex space $E$ into a Fréchet space $F$ has a representation of the form $T x=\sum \lambda_{n}\left\langle x, a_{n}\right\rangle y_{n}$, where $\lambda$ is a sequence in $c_{0},\left\{a_{n}\right\}$ is an equicontinuous sequence in the topological dual $E^{\prime}$ of $E$ and $\left\{y_{n}\right\}$ is an unconditionally summable sequence in $F$, if and only if $T$ can be "compactly factored" through $c_{0}$.
\end{abstract}

A linear operator $T: E \rightarrow F$ from one locally convex space into another is precompact [respectively, compact] if $T$ transforms a neighborhood of 0 in $E$ into a precompact [respectively, relatively compact] subset of $F$. (All of our spaces are assumed to be Hausdorff.)

A sequence $\left\{y_{n}\right\}$ in a sequentially complete locally convex space $E$ is unconditionally summable if $\sum \xi_{n} y_{n}$ converges for each $\xi$ in $l_{\infty}$. $\left(l_{\infty}\right.$ is the Banach space of bounded sequences equipped with the supremum norm $\|\cdot\|_{\infty}$.) For conditions equivalent to unconditional summability we refer the reader to [3, Chapter IV, $\S 1$, pp. 58-59]. A sequence $\left\{y_{n}\right\}$ in a locally convex space $E$ is weakly unconditionally summable if $\sum\left|\left\langle y_{n}, b\right\rangle\right|<\infty$ for each $b$ in the topological dual $E^{\prime}$ of $E$.

Let $q$ be a seminorm on a locally convex space $E$. Let $E(q)=E / q^{-1}(0)$, and let $K_{q}: E \rightarrow E(q)$ denote the natural map. Equip $E(q)$ with the norm $\left\|K_{q}(x)\right\|=q(x)$. Following $[6,1.2]$ we say that $q$ is precompact if the natural map $K_{q}$ is precompact. In $[6,2.5]$ we showed that $q$ is a precompact seminorm on $E$ if and only if there is a sequence $\lambda$ in $c_{0}$ and an equicontinuous sequence $\left\{a_{n}\right\}$ in $E^{\prime}$ such that, for each $x$ in $E$,

$$
q(x) \leqq \sup \left|\lambda_{n}\right|\left|\left\langle x, a_{n}\right\rangle\right| \text {. }
$$

Received by the editors April 15, 1972 and, in revised form, April 27, 1972.

AMS (MOS) subject classifications (1970). Primary 47A65, 47B05; Secondary 46B15.

Key words and phrases. Normed space, Banach space, Fréchet space, compact linear operator, precompact linear operator, unconditionally summable sequence, weakly unconditionally summable sequence, equicontinuous sequence, normalized basis, associated sequence of coefficient forms.

(c) American Mathematical Society 1973 
In $[6,2.10$, p. 92] we showed that a linear operator $T: E \rightarrow F$ from a locally convex space $E$ into a normed space $F$ is precompact if and only if there is a precompact seminorm $q$ on $E$ such that $\|T x\| \leqq q(x)$ for each $x$ in $E$. (In the normed space case this result has also been given by Terzioglu $[9,(1)$, p. 93] and $[8$, Theorem 1, p. 76].)

The following lemma is an immediate consequence of [ 2 , Theorem 2.4 , p. 477].

1. Lemma. A seminorm $q$ on $c_{0}$ is precompact if and only if there is a sequence $\lambda$ in $c_{0}$ such that, for each $\mu$ in $c_{0}, q(\mu) \leqq \sup \left|\lambda_{n}\right|\left|\mu_{n}\right|$.

2. Proposition. A linear operator $T: c_{0} \rightarrow E$ from $c_{0}$ into a normed space $E$ is precompact if and only if there is a sequence $\lambda$. in $c_{0}$ and a sequence $\left\{y_{n}\right\}$ in $E$, that is (weakly) unconditionally summable in the completion of $E$, such that, for each $\mu$ in $c_{0}, T \mu=\sum \lambda_{n} \mu_{n} y_{n}$.

Proof. Lemma 1 and [1, Lemma 2, p. 159] show that the condition is sufficient. We now prove necessity.

Suppose $T: c_{0} \rightarrow E$ is precompact; then (see Lemma 1 ) there is a sequence $\lambda$ in $c_{0}$ such that, for each $\mu$ in $c_{0}$,

$$
\|T \mu\| \leqq \sup \left|\lambda_{n}\right|^{2}\left|\mu_{n}\right|
$$

We assume that each $\lambda_{n} \neq 0$. For each $n$, let $y_{n}=T\left[\left(\lambda_{n}\right)^{-1} e_{n}\right]$ (where $\left\{e_{n}\right\}$ is the usual unit vector basis of $c_{0}$ ). Since

$$
\left\|\sum_{k=n}^{m} \xi_{k} y_{k}\right\|=\left\|T\left[\sum_{k=n}^{m} \xi_{k}\left(\lambda_{k}\right)^{-1} e_{k}\right]\right\| \leqq \sup \left\{\left|\lambda_{k}\right|\left|\xi_{k}\right|: k \geqq n\right\}
$$

for each $\xi$ in $l_{x}$, it follows that $\left\{y_{n}\right\}$ is unconditionally summable in the completion of $E$. Since

$$
\left\|T \mu-\sum_{k=1}^{n} \lambda_{k} \mu_{k} y_{k}\right\|=\left\|T\left[\sum_{k>n} \mu_{k} e_{k}\right]\right\| \leqq \sup \left\{\left|\lambda_{k}\right|^{2}\left|\mu_{k}\right|: k>n\right\}
$$

for each $\mu$ in $c_{0}$, it follows that $T \mu=\sum \lambda_{n} \mu_{n} y_{n}$.

The following theorem is closely related to Theorem 2.18 in [6] and shows that (in the Fréchet space case) the Schwartz maps introduced in $[6,2.15]$ coincide with the linear operators that can be "compactly factored" through $c_{0}$. Recall that a Fréchet space is a complete metrizable locally convex space.

3. THEOREM. Let $T: E \rightarrow F$ be a linear operator from a locally convex space $E$ into a Fréchet space $F$. The following are equivalent:

(a) $T$ has a representation of the form

$$
T x=\sum \lambda_{n}\left\langle x, a_{n}\right\rangle y_{n}
$$




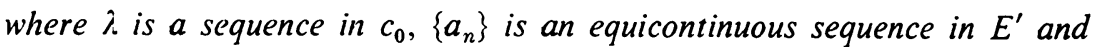
$\left\{y_{n}\right\}$ is an unconditionally summable sequence in $F$.

(b) Same as (a) except that $\left\{y_{n}\right\}$ is weakly unconditionally summable.

(c) There exist compact linear operators $P: E \rightarrow c_{0}$ and $Q: c_{0} \rightarrow F$ such that $T=Q P$.

(d) Same as (c) except that $Q$ is not assumed to be compact, but only continuous.

(e) Same as (c) except that $P$ is not assumed to be compact, but only continuous.

Proof. (a) implies (b) is clear.

(b) implies (d). Suppose that $T x=\sum \lambda_{n}\left\langle x, a_{n}\right\rangle y_{n}$ where $\lambda$ is a sequence in $c_{0},\left\{a_{n}\right\}$ is an equicontinuous sequence in $E^{\prime}$ and $\left\{y_{n}\right\}$ is a weakly unconditionally summable sequence in $F$. By [5, Satz 1.3 .5 , p. 24] it follows that $\sum \mu_{n} y_{n}$ converges in $F$, whenever $\mu$ is a sequence in $c_{0}$. Let $P: E \rightarrow c_{0}$ be defined by $P(x)=\left\{\lambda_{n}\left\langle x, a_{n}\right\rangle\right\}$; and let $Q: c_{0} \rightarrow F$ be defined by $Q(\mu)=$ $\sum \mu_{n} y_{n}$. Then $T=Q P$ and by using [4, III.2,3, p. 85] it is easy to show that $P$ is compact. (Since $\left\{a_{n}\right\}$ is equicontinuous, $P$ is continuous.)

(d) implies (c) follows easily from [6, Theorem 2.18] and the fact that $c_{0}$ has the $c_{0}$-extension property (see $[6,2.17]$ ).

(c) implies (e) is clear.

(e) implies (a) follows from Proposition 2, [6, 2.11] and [6, 2.19].

4. REMARK. Compact linear operators having representations of the form described in Theorem 3(a) have been studied by Terzioglu [8] and [9], and independently by the author [6]. By using the results of [6] it is easy to see that Theorem 3 remains valid if the hypothesis, that $F$ be a Fréchet space, is replaced by the weaker hypothesis that $F$ be a sequentially complete locally convex space satisfying any one of the conditions of [6, 2.12]. By modifying the proof of Proposition 2 we can show that (given $\varepsilon>0$ ) every compact linear operator $T: c_{0} \rightarrow E$ from $c_{0}$ into a Banach space $E$ has a representation of the form described in Theorem 3 with $\|\lambda\|_{\infty} \leqq$ $\|T\|+\varepsilon$ and $\left\|\sum \xi_{n} y_{n}\right\| \leqq\|\xi\|_{\infty}$ for all $\xi$ in $l_{\infty}$.

We now show that the condition in Proposition 2 actually characterizes the Banach space $c_{0}$. Recall that $\left\{x_{n}\right\}$ is a normalized basis for a Banach space $E$ if $\left\|x_{n}\right\|=1$ and each $x$ in $E$ has a unique representation of the form $x=\sum \alpha_{n} x_{n}$. The sequence $\left\{f_{n}\right\}$ of linear forms defined by $x=\sum\left\langle x, f_{n}\right\rangle x_{n}$ is called the associated sequence of coefficient forms. It is well known (see [7, Theorem 3.1, p. 20]) that $\sup \left\|f_{n}\right\|<\infty$.

Let $\left\{x_{n}\right\}$ and $\left\{y_{n}\right\}$ be normalized bases for Banach spaces $E$ and $F$, respectively. The bases $\left\{x_{n}\right\}$ and $\left\{y_{n}\right\}$ are equivalent if for each sequence $\xi$ in $l_{\infty}$ the series $\sum \xi_{n} x_{n}$ converges if and only if the series $\sum \xi_{n} y_{n}$ converges. If $\left\{x_{n}\right\}$ is equivalent to $\left\{y_{n}\right\}$, then (see $[1,1.2$, p. 152]) $E$ and $F$ are 
isomorphic Banach spaces. In fact, (see [7, Theorem 8.1(d), pp. 69-70]) the function $T\left(x_{n}\right)=y_{n}$ can be extended to a continuous linear operator from $E$ onto $F$.

5. THEOREM. Let $E$ be a Banach space with normalized basis $\left\{x_{n}\right\}$. Let $\left\{f_{n}\right\}$ denote the associated sequence of coefficient forms. The following are equivalent:

(a) $\left\{x_{n}\right\}$ is equivalent to the unit vector basis of $c_{0}$.

(b) For each compact linear operator $T: E \rightarrow F$ from $E$ into a Banach space $F$, there is a sequence $\lambda$ in $c_{0}$ and an unconditionally summable sequence $\left\{y_{n}\right\}$ in $F$ such that, for each $x$ in $E, T x=\sum \lambda_{n}\left\langle x, f_{n}\right\rangle y_{n}$.

(c) Same as (b) except that $\left\{y_{n}\right\}$ is weakly unconditionally summable.

(d) For each compact linear operator $T: E \rightarrow F$ from $E$ into a Banach space $F$, there is a sequence $\lambda$ in $c_{0}$ such that, for each $x$ in $E$,

$$
\|T x\| \leqq \sup \left|\lambda_{n}\right|\left|\left\langle x, f_{n}\right\rangle\right|
$$

(e) For each precompact seminorm $q$ on $E$ there is a sequence $\lambda$ in $c_{0}$ such that, for each $x$ in $E$,

$$
q(x) \leqq \sup \left|\lambda_{n}\right|\left|\left\langle x, f_{n}\right\rangle\right|
$$

Proof. (a) implies (b) follows from Proposition 3. (b) implies (c) is trivial. (c) implies (d) is an easy consequence of [1, Lemma 2, p. 159]. (d) implies (e) follows from the fact that $q(x)=\left\|K_{q}(x)\right\|$ (see the introduction).

(e) implies (a). By [1, Lemma 3, p. 160] it suffices to show that $\sum\left|\left\langle x_{n}, a\right\rangle\right|<\infty$ for each $a$ in $E^{\prime}$. Let $a$ be an element of $E^{\prime}$; then (by (e)) there is a sequence $\lambda$ in $c_{0}$ such that for each $x$ in $E$

$$
|\langle x, a\rangle| \leqq \sup \left|\lambda_{n}\right|\left|\left\langle x, f_{n}\right\rangle\right|
$$

For each $n$ let $\alpha_{n}$ be a scalar such that $\alpha_{n}\left\langle x_{n}, a\right\rangle=\left|\left\langle x_{n}, a\right\rangle\right|$ and $\left|\alpha_{n}\right|=1$. Let $x=\alpha_{1} x_{1}+\cdots+\alpha_{k} x_{k}$; then (by $(*)$ )

$$
\left|\left\langle x_{1}, a\right\rangle\right|+\cdots+\left|\left\langle x_{k}, a\right\rangle\right|=|\langle x, a\rangle| \leqq \sup \left|\lambda_{n}\right| M,
$$

where $M=\sup \left\|f_{n}\right\|$. Therefore, $\sum\left|\left\langle x_{n}, a\right\rangle\right|<\infty$ for $a$ in $E^{\prime}$ and $\left\{x_{n}\right\}$ is equivalent to the unit vector basis of $c_{0}$.

ADDED IN PROOF. It has recently been brought to our attention that in the Banach space case Grothendieck (using his theory of tensor products) has essentially proven Proposition 2. See Sur certaines classes de suites dans les espaces de Banach, et le théorème de Dvoretzky-Rogers, Bol. Soc. Mat. São Paulo 8 (1953), 81-110 (page 88). 


\section{REFERENCES}

1. C. Bessaga and A. Pelczyński, On bases and unconditional convergence of series in Banach spaces, Studia Math. 17 (1958), 151-164. MR 22 \#5872.

2. J. B. Conway, The strict topology and compactness in the space of measures. II, Trans. Amer. Math. Soc. 126 (1967), 474-486. MR 34 \#6503.

3. M. M. Day, Normed linear spaces, 2nd ed., Ergebnisse der Mathematik und ihrer Grenzgebiete, N.F., Heft 21, Academic Press, New York; Springer-Verlag, Berlin, 1962. MR 26 \#2847.

4. S. Goldberg, Unbounded linear operators: Theory and applications, McGraw-Hill, New York, 1966. MR 34 \#580.

5. A. Pietsch, Nukleare lokalkonvexe Räume, 2nd ed., Akademie-Verlag, Berlin, 1969.

6. D. Randtke, Characterizations of precompact maps, Schwartz spaces and nuclear spaces, Trans. Amer. Math. Soc. 165 (1972), 87-101.

7. I. Singer, Bases in Banach spaces. I, Springer-Verlag, New York, 1970.

8. T. Terzioglu, A characterization of compact linear mappings, Arch. Math. 22 (1971), 76-78.

9. - On compact and infinite-nuclear mappings, Bull. Math. Soc. Sci. Math. R.S. Roumanie 14 (62) (1970), 93-99.

Department of Mathematics, University of Georgia, Athens, Georgia 30601 